\title{
Mechanical Fault Diagnosis for HV Circuit Breakers Based on Ensemble Empirical Mode Decomposition Energy Entropy and Support Vector Machine
}

\author{
Jianfeng Zhang, ${ }^{1}$ Mingliang Liu, ${ }^{1,2}$ Keqi Wang, ${ }^{2}$ and Laijun Sun ${ }^{1}$ \\ ${ }^{1}$ HLJ Province Key Lab of Senior-Education for Electronic Engineering, Heilongjiang University, Harbin 150080, China \\ ${ }^{2}$ College of Mechanical and Electrical Engineering, Northeast Forestry University, Harbin 150040, China \\ Correspondence should be addressed to Mingliang Liu; mll_0608@163.com
}

Received 8 April 2015; Revised 19 June 2015; Accepted 28 June 2015

Academic Editor: Jean-Charles Beugnot

Copyright (C) 2015 Jianfeng Zhang et al. This is an open access article distributed under the Creative Commons Attribution License, which permits unrestricted use, distribution, and reproduction in any medium, provided the original work is properly cited.

During the operation process of the high voltage circuit breaker, the changes of vibration signals can reflect the machinery states of the circuit breaker. The extraction of the vibration signal feature will directly influence the accuracy and practicability of fault diagnosis. This paper presents an extraction method based on ensemble empirical mode decomposition (EEMD). Firstly, the original vibration signals are decomposed into a finite number of stationary intrinsic mode functions (IMFs). Secondly, calculating the envelope of each IMF and separating the envelope by equal-time segment and then forming equal-time segment energy entropy to reflect the change of vibration signal are performed. At last, the energy entropies could serve as input vectors of support vector machine (SVM) to identify the working state and fault pattern of the circuit breaker. Practical examples show that this diagnosis approach can identify effectively fault patterns of HV circuit breaker.

\section{Introduction}

As an import part of the electric system, a HV circuit breaker is a key device to control and protect the power network. Therefore, the action reliability of HV circuit breaker is extremely important in the electric system. In recent years, research on diagnosis method of circuit breaker is growing fast, and many new techniques have been used in practice, in which the technique based on the analysis of the vibration signal has gradually become hot [1-3].

Many vibration signals produced by circuit breaker contain a number of pieces of important information, which can be used to evaluate the machinery state of circuit breaker. Through the analysis of vibration signals acquired by the piezoelectric sensor, the running states of circuit breaker are convenient and accurate to diagnose. To analyze the vibration signal, some signal processing methods, such as wavelet $[4,5]$ and EMD [6, 7], have been used in practice. Wavelet analysis has become popular in the past decade as a method for time-frequency representation. In principle, wavelet transform (WT) uses short windows at high frequencies and long windows at low frequencies, which renders WT more suitable for dealing with nonstationary time series. Nonetheless, wavelet analysis is also limited by the fundamental uncertainty principle, in which both time and frequency cannot simultaneously be resolved with the same precision. Moreover, the results of WT analysis depend on the choice of the mother wavelet, which is arbitrary and may not be optimal for the time series under scrutiny. In contrast to the WT approach, the empirical mode decomposition (EMD) [8] method adaptively decomposes nonstationary time series into narrow-band components, namely, intrinsic mode functions (IMFs), by empirically identifying the physical time scales intrinsic to the data without assuming any basis functions. Thus, the EMD can potentially localize events in both time and frequency, even in nonstationary time series [9-12]. So, the EMD is a suitable method to process nonlinear and nonstationary signals. However, mode mixing problems brought by EMD greatly restrict its application in practice. EEMD is the repeated EMD by adding Gauss white noise in each of the decompositions. It takes advantage of the uniform 
distribution statistical characteristics of Gauss white noise in frequency domain [13]. Through this method, EEMD could decompose signal continuously in different scales. Therefore, the problem of mode mixing will be eliminated effectively. A nonstationary vibration signal is decomposed into a series of intrinsic mode functions (IMFs) by EEMD. The envelope of IMF can be obtained through Hilbert transform and separated by equal-time segment. Then, we can get the energy entropy of each envelope of IMF with the energy entropy theory. Those IMF energy entropies can form the entropy vector, and this could serve as the input vector of SVM for judging circuit breaker working states and fault types. The experiment result indicates the method that applied the EEMD-energy entropy and support vector machine is effective and has many potential applications in practice.

\section{EEMD Method}

EEMD is a new method of signal process; the specific decomposition steps and principles are as follows [14].

Step 1. Adding the random Gauss white noise $n_{i}(t)$ with the mean zero of amplitude and the constant of standard deviation to the original signal $x(t)$ (the standard deviation of white noise is $0.1-0.4$ times the size of the original signal.), the function is as follows:

$$
x_{i}(t)=x(t)+n_{i}(t) .
$$

Signal $x_{i}(t)$ is the signal that added the $i$ th Gauss white noise. The Gauss white noise will directly affect the decomposition of signal by EEMD.

Step 2. Signal $x_{i}(t)$ is decomposed into several IMFs $c_{i j}(t)$ and the margin $r_{i}(t)$. The $c_{i j}(t)$ with the $i$ th Gauss white noise is the $j$ th IMF decomposition.

Step 3. Repeat Steps 1 and $2 N$ times. Next, with the principle that the statistical mean of random and independent sequence is zero, the overall average operation for the corresponding IMF could eliminate the effects of multiple Gauss white noise on the real IMF. The final IMF is written as

$$
c_{j}(t)=\frac{1}{N} \sum_{i=1}^{N} c_{i j}
$$

in which the $c_{j}$ is the $j$ th IMF component of original signal by EEMD. When $N$ is larger, the sum of the white noise of IMFS will tend to zero. At this time, the results for EEMD are written as

$$
x(t)=\sum_{j} c_{j}(t)+r(t),
$$

in which $r(t)$ is the final residual component, representing the average trend of signal. Through EEMD method, we can put any signal $x(t)$ into several of IMFs and a residual component. The intrinsic mode components $c_{j}(t)(j=1,2, \ldots)$ represent the elements of signal from high to low frequency band; in each band the frequency components are not the same and will change following the change of vibration signal $x(t)$.

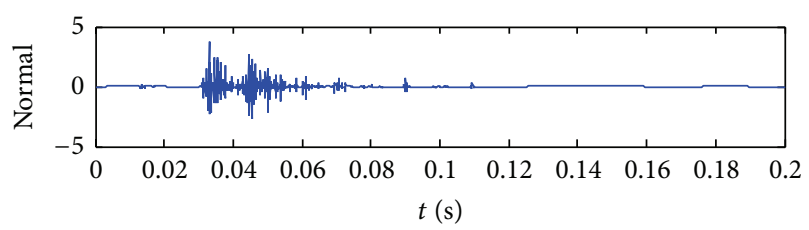

FIgURE 1: Standard signal of normal state.
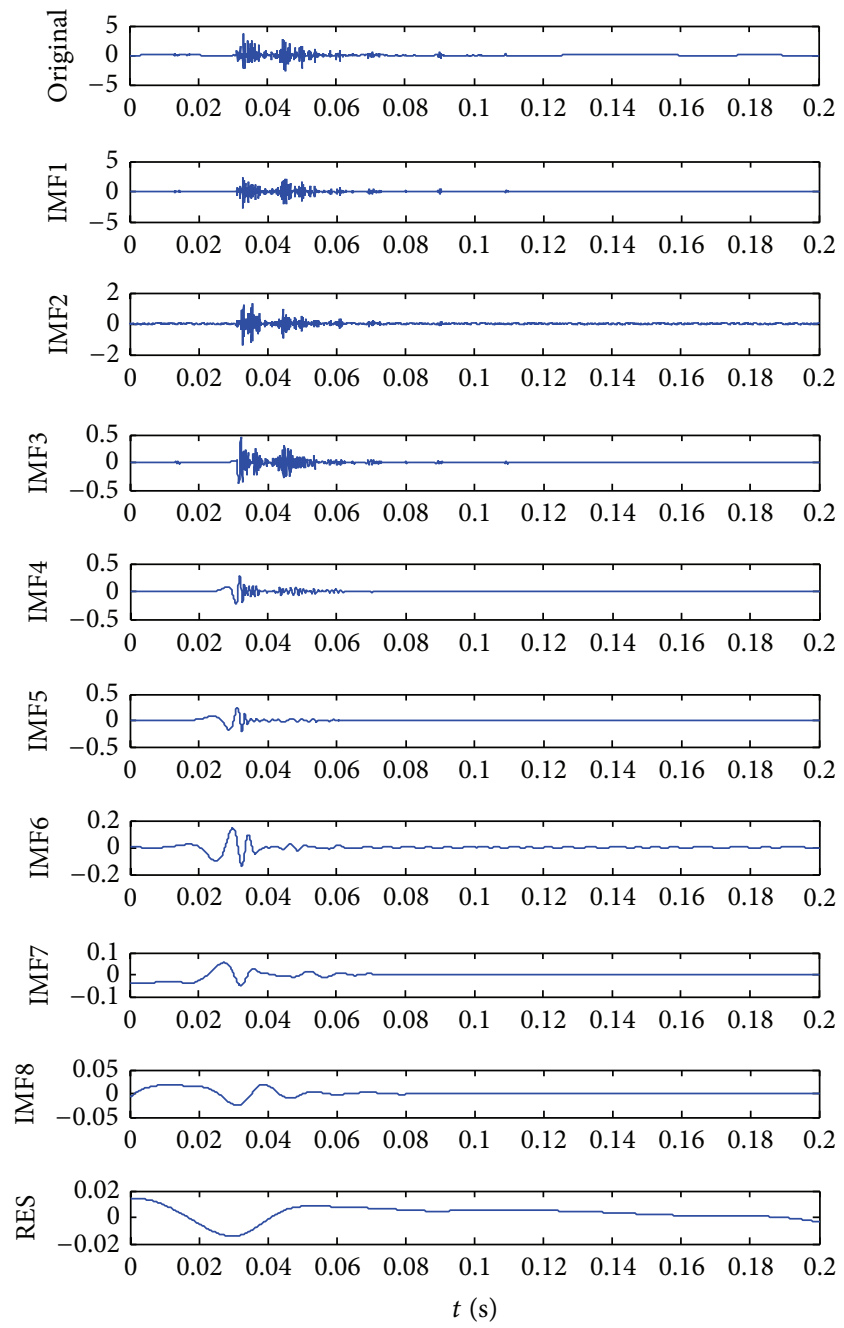

FIgURE 2: Results of EEMD decomposition.

Figure 1 shows the normal state of vibration signal. The signal can get eight major components and a residual component by EEMD, as shown in Figure 2. From the diagram, the normal state of nonstationary vibration signal is decomposed into a number of stationary IMF components by EEMD, and different IMF component contains a variety of time scales.

\section{EEMD-Energy Entropy}

3.1. The Extraction Envelope of the Signal. Mutation information of signal is often presented in the envelope of the signal. When the operating mechanism of circuit breaker is in 
action, the high frequency components from the impact can be seen as a signal carrier of the envelope signal. Therefore, the Hilbert method which is used to extract the envelope of signal for diagnosis is very effective in the mechanical fault diagnosis.

For a real signal $x(t)$, the Hilbert transform is defined as

$$
\widehat{x}(t)=\frac{1}{\pi t} \cdot x(t)=\frac{1}{\pi} \int_{-\infty}^{+\infty} \frac{x(\tau)}{t-\tau} d \tau .
$$

Then, the analytic signal of $x(t)$ is

$$
g(t)=x(t)+j \widehat{x}(t) .
$$

The amplitude of $g(t)$ is

$$
A(t)=\sqrt{x^{2}(t)+\widehat{x}^{2}(t)} .
$$

So $A(t)$ is the envelope of $x(t)$.

3.2. The Application of Entropy. Entropy is an information measure for describing the complexity or irregularity of system. So far, many attempts have been made for estimation of entropy in the complex system, such as Kolmogorov entropy, approximate entropy, and Shannon information entropy [15]. This paper chooses the Shannon information entropy. The information entropy can reflect the uniformity of probability distribution of system. The greater the entropy value $H$, the more uniform the information distribution and the greater the disorder degree of the information. Therefore, it can also be used to describe the uncertainty degree of the system [16$18]$.

Let an information system have $N$ random information sources $x_{1}, x_{2}, \ldots, x_{N}$, and the probability of each information source appearing in the whole system is $p_{1}, p_{2}, \ldots, p_{N}$, respectively. Then, its information entropy is defined as follows:

$$
H=-\sum_{i=1}^{N} p_{i} \log p_{i} .
$$

Distinguishing normal state and fault state is the essence of fault diagnosis of circuit breaker. The fault can be regarded as different mutations in the normal state. According to this property, this paper proposes the equal-time segment approach to achieve extraction of entropy. The principle is shown in Figure 3.

In Figure 3, Signal 1 is the envelope of normal signal. Signal 2 is the envelope of fault signal. Mutation events are delayed. Signal 2 was compared with Signal 1. Signal 1 was segmented according to equal-time segment. Each of them has three segments: Seg1, Seg2, and Seg3. Because Signal 2 is changed compared with Signal 1, Seg1, Seg2, and Seg3 energies of Signal 2 compared with Seg1, Seg2, and Seg3 energies of Signal 1 are also changed, indicating that energy distribution is changed. Therefore, we can transform the changes of Signal 1 and Signal 2 into the change of energy distribution uniformity of each segment.

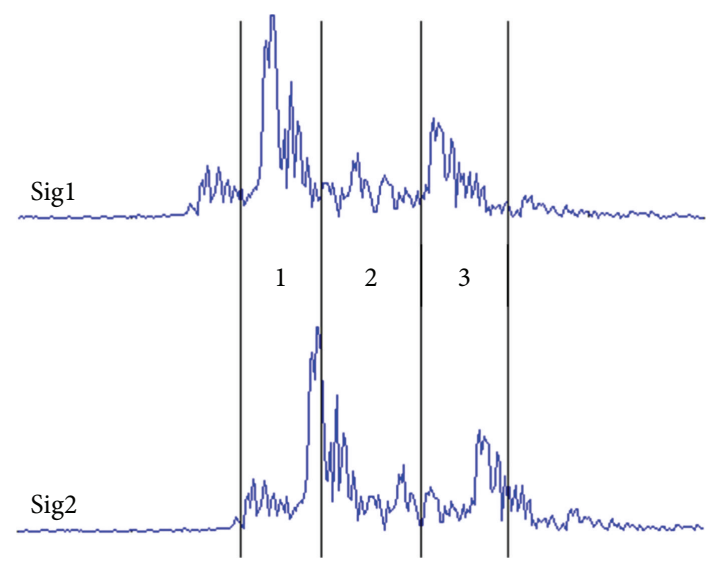

Figure 3: Segments with equal-time.

3.3. The Extraction Steps of Entropy. With the extraction of EEMD-energy entropy of sampling vibration signal as an example, the specific extraction steps are as follows.

Step 1. First vibration signal is denoised by wavelet soft threshold.

Step 2. The denoising signal is decomposed by EEMD and choosing the top 8 main intrinsic mode functions (IMFs).

Step 3. Get the respective analytic signals of the obtained IMFs with Hilbert transform.

Step 4. Extract the envelope of the respective analytic signals.

Step 5. Separate equally the envelope of signal into $M$ sections along the time axis, and calculate the energy of each segment according to the following equation:

$$
Q_{k}(i)=\int_{t_{1}}^{t_{2}}|A(t)|^{2} d t
$$

in which $i=1,2, \ldots, M, k=1,2, \ldots, 8$, and $t_{1}$ and $t_{2}$ are the starting and stopping time of the $i$ segment.

Step 6. Normalization processing is made to the signal envelope of the segmented energy as follows:

$$
q_{k}(i)=\frac{Q_{k}(i)}{\sum_{i=1}^{m} Q_{k}(i)} .
$$

Step 7. According to the basic theory of entropy, the definition of the EEMD-energy entropy for the envelope of signal $A(t)$ is written as

$$
H_{k}=-\sum_{i=1}^{m} q_{k}(i) \lg q_{k}(i) .
$$

Step 8. Finally, the EEMD-energy entropy vector is

$$
T=\left[H_{1}, H_{2}, \ldots, H_{8}\right] \text {. }
$$


When we use the method of the EEMD-energy entropy for fault detection, actually, the distribution of normal signals is considered to be as a uniform distribution. Moreover, the distribution of the test signals under fault condition is not uniform. And because the entropy is a measure of signal heterogeneity degree, we can use the EEMD-energy entropy to reflect the deviation degree of fault state relative to the normal state.

\section{SVM}

SVM is a promising classifier that minimizes the empirical classification error and at the same time maximizes the margin by determining a separating hyperplane to distinguish different classes of data [19]. The basic idea of support vector machine is to create a hyperplane as the optimal separating hyperplane. The optimal separating hyperplane can not only make all samples correct classification but also have the maximum distance to the nearest point of the training data. To enable the optimal separating hyperplane method to be generalised, Cortes and Vapnik introduced nonnegative variable $\varepsilon_{i}$ and the penalty factor $C$. The $\varepsilon_{i}$ are a measure of the misclassification errors and the $C$ is a given value and subject to the constraints of (12). Then, the constraint conditions of the hyperplane are written as

$$
y_{i}[(\omega \cdot x)+b] \geq 1-\varepsilon_{i} \quad i=1,2, \ldots, l .
$$

Hence, the optimal separating hyperplane is given by

$$
\phi(\omega, \varepsilon)=\frac{1}{2} \omega \cdot \omega+c \sum_{i=1}^{l} \varepsilon_{i} .
$$

By introducing the kernel function $K\left(x_{i} \cdot x_{j}\right)$, the nonlinear problem could be transformed into a linear problem in high dimension space. The corresponding decision function is written as

$$
f(x)=\operatorname{sgn}\left[\sum_{\mathrm{SV}} a_{i} y_{i} K\left(x_{i} \cdot x_{j}\right)+b\right] .
$$

The classification performance of SVM is superior to the neural network classifier in the fault diagnosis for small samples. Put $T$ as the input vector of SVM, choose the radial basis function $(\mathrm{RBF})$, and use the strategy of "hierarchical SVMs (H-SVMs)" $[20,21]$ for the mechanical fault diagnosis of circuit breakers.

H-SVMs have a variety of classification structures; this research chooses the skewed tree classification structure; its structure is shown in Figure 4. H-SVMs classify the four kinds of states that are the normal state $\mathrm{Cl}$, the lack of lubrication state $\mathrm{C} 2$, the foundation bolt looseness state $\mathrm{C} 3$, and the energy storage spring shed state $\mathrm{C} 4$ into three levels of classification training and recognition by three SVMs. First, using a SVM to the first level classification, C4 is separated from $\mathrm{C} 1, \mathrm{C} 2$, and $\mathrm{C} 3$; the second stage is separating $\mathrm{C} 3$ from $\mathrm{C} 1$ and $\mathrm{C} 2$ by the second SVM; finally, the third SVM is employed to the third grade classification, separating $\mathrm{Cl}$ and C2.

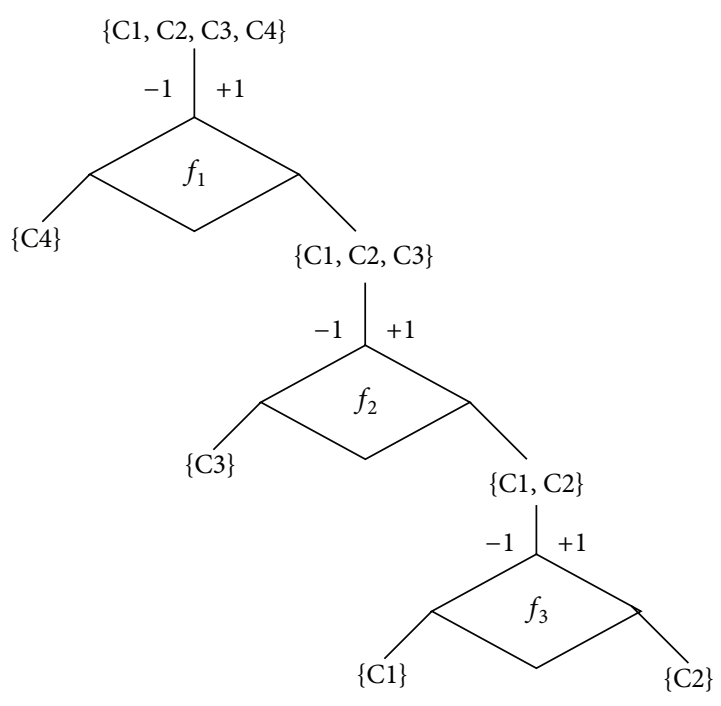

FIGURE 4: Classification tree diagram for H-SVMs.
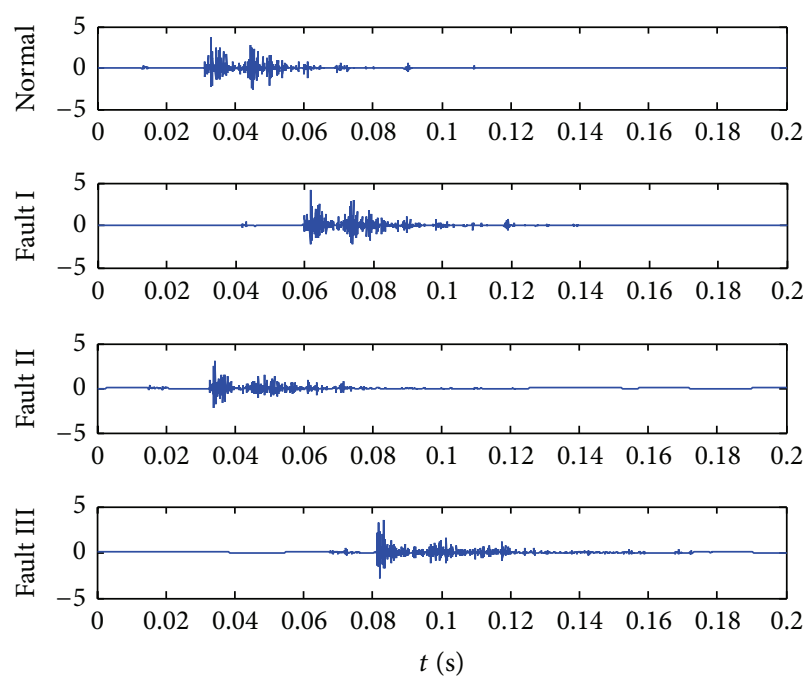

FIGURE 5: Vibration signals of vacuum circuit breaker.

\section{Experiment and Analysis}

Collecting the vibration signals of the normal state, the lack of lubrication state, the foundation bolt looseness state, and the energy storage spring shed state from type ZW32-12 of vacuum circuit breaker in laboratory, as shown in Figure 5, each state collected 20 groups of close-brake vibration signal, including 10 groups for SVM training and 10 groups for testing. Adopting the method of EEMD-energy entropy to calculate each state of the close-brake vibration signals, getting the 8-dimensional entropic vector $T$ of each sample, a part of the entropy values is shown in Table 1.

Then, the received entropic vectors are inputted $\mathrm{H}$ SVMs for classification and recognition. Radial basis kernel function is applied in this experiment. Penalty factor $C$ and the kernel function parameter $g$ are two important parameters for influencing the accuracy and generalization 
TABLE 1: Vectors of characteristic entropy.

\begin{tabular}{|c|c|c|c|c|c|c|c|c|}
\hline & $H_{1}$ & $\mathrm{H}_{2}$ & $\mathrm{H}_{3}$ & $\mathrm{H}_{4}$ & $\mathrm{H}_{5}$ & $H_{6}$ & $\mathrm{H}_{7}$ & $H_{8}$ \\
\hline Normal & 1.38995 & 1.2863 & 1.31565 & 1.22686 & 1.19784 & 1.08202 & 1.1478 & 1.35075 \\
\hline Normal & 1.3758 & 1.37933 & 1.32379 & 1.18654 & 1.19728 & 1.01396 & 1.18778 & 1.32624 \\
\hline Normal & 1.42401 & 1.2713 & 1.32004 & 1.25894 & 1.23718 & 1.17143 & 0.97525 & 1.39509 \\
\hline Fault I & 0.89786 & 0.92083 & 0.97844 & 1.11754 & 0.96972 & 1.20403 & 1.45528 & 1.89058 \\
\hline Fault I & 0.92882 & 0.86088 & 0.91616 & 0.92303 & 1.03207 & 1.19891 & 1.58584 & 1.85211 \\
\hline Fault I & 0.83574 & 0.91725 & 0.96161 & 1.19681 & 1.03097 & 1.23316 & 1.50922 & 2.05601 \\
\hline Fault II & 1.59699 & 1.3049 & 1.19265 & 0.90063 & 0.96383 & 1.00915 & 1.02713 & 1.67725 \\
\hline Fault II & 1.57129 & 1.28567 & 1.22408 & 1.06797 & 0.97543 & 0.97423 & 0.95939 & 1.66329 \\
\hline Fault II & 1.54017 & 1.24947 & 1.23603 & 1.03721 & 1.09331 & 1.0717 & 1.07941 & 1.76371 \\
\hline Fault III & 1.13835 & 0.98421 & 1.08779 & 1.09961 & 1.37122 & 1.49651 & 1.93909 & 2.07203 \\
\hline Fault III & 1.16172 & 1.02966 & 0.85995 & 0.99001 & 1.32936 & 1.521 & 1.93032 & 1.94298 \\
\hline Fault III & 1.19095 & 0.81114 & 1.04993 & 1.02732 & 1.31641 & 1.45068 & 1.93723 & 2.04867 \\
\hline
\end{tabular}

TABLE 2: Recognition results of H-SVMs.

\begin{tabular}{lcc}
$\begin{array}{l}\text { The type of } \\
\text { state }\end{array}$ & Recognition results & Accuracy/\% \\
\hline $\begin{array}{l}\text { Normal } \\
\text { Fault I }\end{array}$ & $\mathrm{C} 1, \mathrm{C} 1, \mathrm{C} 1, \mathrm{C} 1, \mathrm{C} 1, \mathrm{C} 1, \mathrm{C} 1, \mathrm{C} 2, \mathrm{C} 2, \mathrm{C} 1, \mathrm{C} 2, \mathrm{C} 2, \mathrm{C} 2, \mathrm{C} 2, \mathrm{C} 2, \mathrm{C} 2$ & 100 \\
Fault II & $\mathrm{C} 3, \mathrm{C} 3, \mathrm{C} 3, \mathrm{C} 3, \mathrm{C} 3, \mathrm{C} 1, \mathrm{C} 3, \mathrm{C} 3, \mathrm{C} 3, \mathrm{C} 3$ & 90 \\
Fault III & $\mathrm{C} 4, \mathrm{C} 4, \mathrm{C} 4, \mathrm{C} 4, \mathrm{C} 4, \mathrm{C} 4, \mathrm{C} 4, \mathrm{C} 4, \mathrm{C} 4, \mathrm{C} 4$ & 100 \\
\hline
\end{tabular}

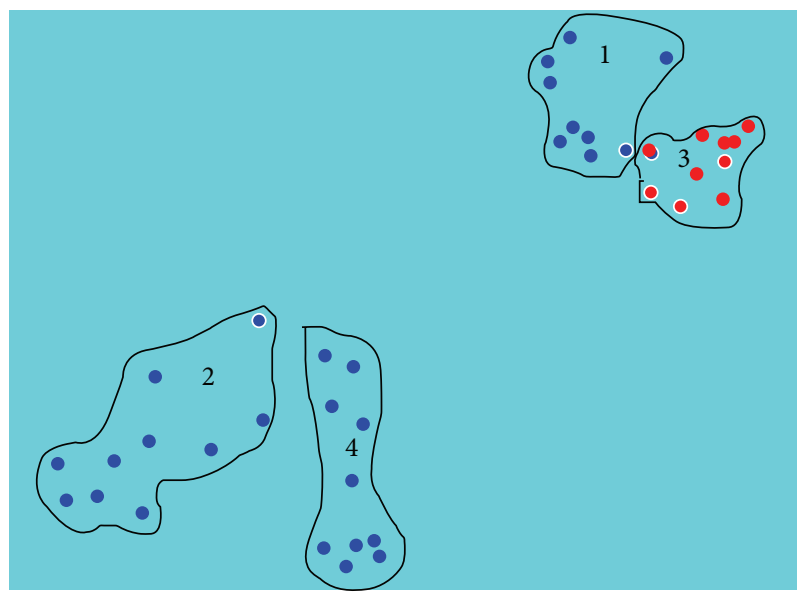

Figure 6: Training results of H-SVMs.

ability of SVM diagnosis. Common methods of parameter optimization are genetic algorithm, grid method, and particle swarm method. This experiment selects the genetic algorithm to determine the optimal parameters $C$ and $g$. Training results are shown in Figure 6, and recognition results are shown in Table 2 . We could see the four training signals are clearly classified from Figure 6 and the accuracy of recognition results is very high from Table 2 in which three types of accuracy are a hundred percent.

\section{Conclusion}

The change of vibration signal of circuit breaker running can reflect circuit breaker operation state. The paper presented an equal-time segment approach based on the EEMD which could influence the change of time, frequency, and energy. Besides, the SVM is effective in solving the circuit breaker status recognition problems that include small samples, nonlinear and high dimension problems. Experimental results show that a combination method of the EEMD, entropy, and SVM for circuit breaker diagnosis to typical fault and normal state has better diagnosis effect.

\section{Conflict of Interests}

The authors confirm that this paper's content has no conflict of interests.

\section{Acknowledgments}

This work is supported by the Natural Science Foundation of Heilongjiang Province of China (E201233) and the Science and Technology Innovative Research Team in Higher Educational Institutions of Heilongjiang Province (no. 2012TD007).

\section{References}

[1] X. Lin, Y.-X. Li, Y.-Q. Ma, and G. Wu, "Dynamic characteristics analysis on novel motor actuator of high voltage circuit breaker," Electric Machines and Control, vol. 13, no. 2, pp. 216-226, 2009.

[2] A. A. Polycarpou, A. Soom, V. Swarnakar et al., "Event timing and shape analysis of vibration bursts from power circuit breakers," IEEE Transactions on Power Delivery, vol. 11, no. 2, pp. 848-857, 1996.

[3] J. Huang, X.-G. Hu, Y.-N. Gong, and F. Yang, "Machinery fault diagnosis expert system for high voltage circuit breaker," Electric Machines and Control, vol. 15, no. 10, pp. 43-49, 2011.

[4] J. Li, "New signal process scheme based on wavelet for online monitoring of high voltage circuit breaker," Journal of Beijing Institute of Machinery, vol. 22, no. 1, pp. 19-22, 2007. 
[5] X. G. Hu, J. Z. Wang, Y. C. Ji et al., "The application of the wavelet analysis of analytic signals in mechanical fault diagnosis of circuit breakers," in Proceedings of the IEEE Power Engineering Society General Meeting, vol. 4, pp. 2235-2240, IEEE/PES, Toronto, Canada, July 2003.

[6] Q. Ma, M. Z. Rong, and S. L. Jia, "Study of switching synchronization of high voltage breakers based on the wavelet packets extraction algorithm and short time analysis method," Proceedings of the Chinese Society for Electrical Engineering, vol. 25, no. 13, pp. 149-154, 2005.

[7] C. Lu and X. G. Hu, "A new method of fault diagnosis for highvoltage circuit-breakers based on Hilbert-Huang transform," in Proceedings of the 2nd IEEE Conference on Industrial Electronics and Applications (ICIEA '07), pp. 2697-2701, IEEE, Harbin, China, May 2007.

[8] N. E. Huang, Z. Shen, S. R. Long et al., "The empirical mode decomposition and the Hubert spectrum for nonlinear and non-stationary time series analysis," Proceedings of the Royal Society A: Mathematical, Physical and Engineering Sciences, vol. 454, no. 1971, pp. 903-995, 1998.

[9] H. Liang, Z. Wang, A. Maier, and N. K. Logothetis, "Single-trial classification of bistable perception by integrating empirical mode decomposition, clustering, and support vector machine," EURASIP Journal on Advances in Signal Processing, vol. 2008, Article ID 592742, 2008.

[10] H. Liang and Z. Lin, "Stimulus artifact cancellation in the serosal recordings of gastric myoelectric activity using wavelet transform," IEEE Transactions on Biomedical Engineering, vol. 49, no. 7, pp. 681-688, 2002.

[11] Z. Wang, A. Maier, D. A. Leopold, N. K. Logothetis, and H. Liang, "Single-trial evoked potential estimation using wavelets," Computers in Biology and Medicine, vol. 37, no. 4, pp. 463-473, 2007.

[12] H. Liang, S. L. Bressler, E. A. Buffalo, R. Desimone, and P. Fries, "Empirical mode decomposition of field potentials from macaque V4 in visual spatial attention," Biological Cybernetics, vol. 92, no. 6, pp. 380-392, 2005.

[13] Z. H. Wu and N. E. Huang, "Ensemble empirical mode decomposition: a noise-assisted data analysis method," Advances in Adaptive Data Analysis, vol. 1, no. 1, pp. 1-41, 2009.

[14] L. Chen, Y. Zi, Z. He, and W. Cheng, "Research and application of ensemble empirical mode decomposition principle and 1.5 dimension spectrum method," Journal of Xian Jiaotong University, vol. 43, no. 5, pp. 94-98, 2009.

[15] M. Hu and H. Liang, "Intrinsic mode entropy based on multivariate empirical mode decomposition and its application to neural data analysis," Cognitive Neurodynamics, vol. 5, no. 3, pp. 277-284, 2011.

[16] R. Rakoczy, M. Kordas, G. Story, and M. Konopacki, "The characterization of the residence time distribution in a magnetic mixer by means of the information entropy," Chemical Engineering Science, vol. 105, no. 2, pp. 191-197, 2014.

[17] H. Su, J. Hu, and Z. Wen, "Structure analysis for concretefaced rockfill dams based on information entropy theory and finite element method," International Journal for Numerical and Analytical Methods in Geomechanics, vol. 36, no. 8, pp. 10411055, 2012.

[18] Y. L. Li, R. P. Shao, and J. M. Cao, "A new and effective method of gear fault diagnosis using wavelet packet transform combined with support vector machine," Journal of Northwestern Polytechnical University, vol. 28, no. 4, pp. 530-535, 2010.
[19] H. Liang and Z. Lin, "Detection of delayed gastric emptying from electrogastrograms with support vector machine," IEEE Transactions on Biomedical Engineering, vol. 48, no. 5, pp. 601604, 2001.

[20] Y. Y. Meng and X. Y. Liu, "A new SVM multi-class classification based on binary tree," Computer Applications, vol. 25, no. 11, pp. 2653-2657, 2005.

[21] Z. G. Yan and P. J. Du, "Construction methods for H-SVMs," Journal of Southeast University, vol. 39, no. 1, pp. 204-209, 2009. 


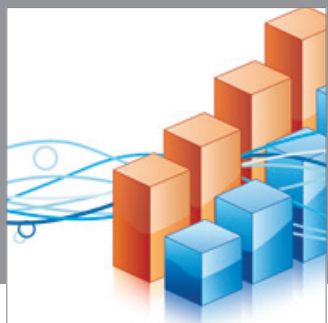

Advances in

Operations Research

mansans

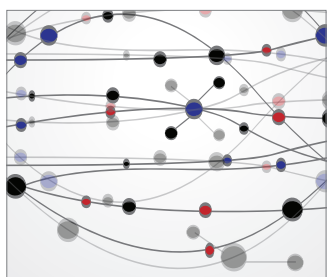

The Scientific World Journal
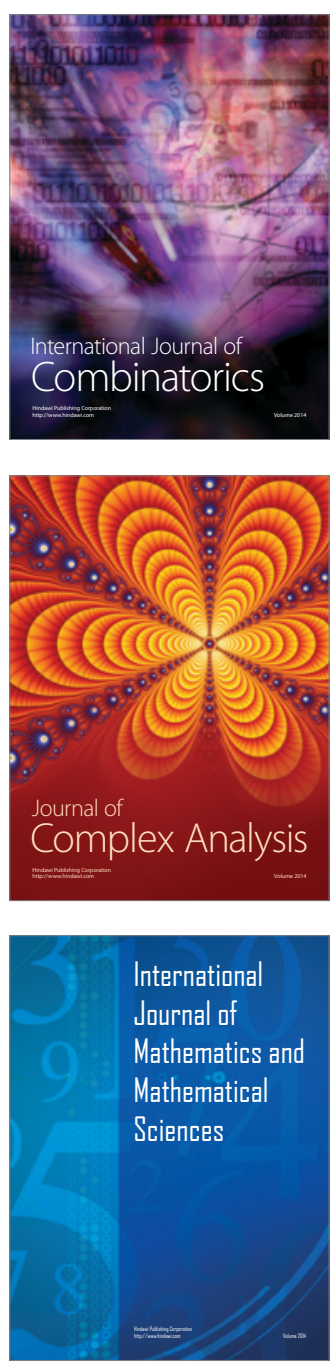
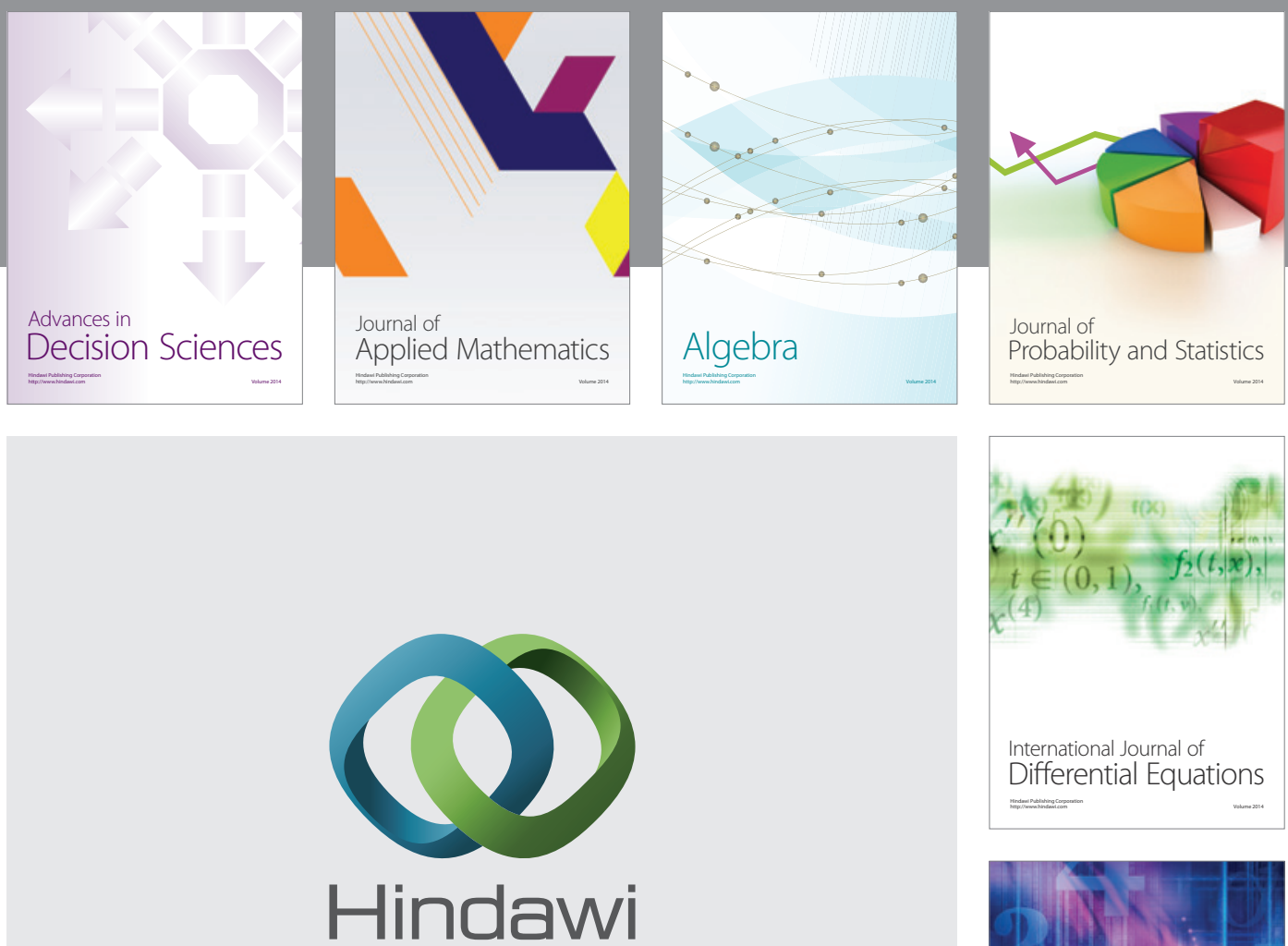

Submit your manuscripts at http://www.hindawi.com
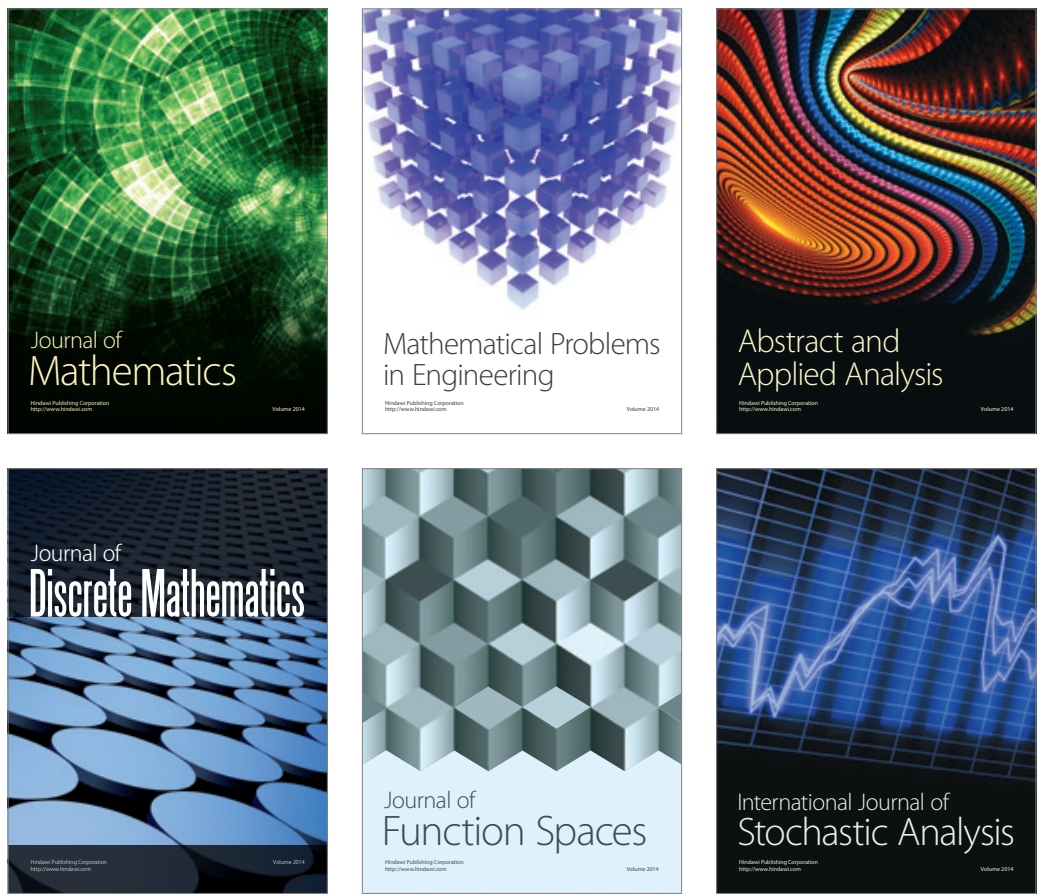

Journal of

Function Spaces

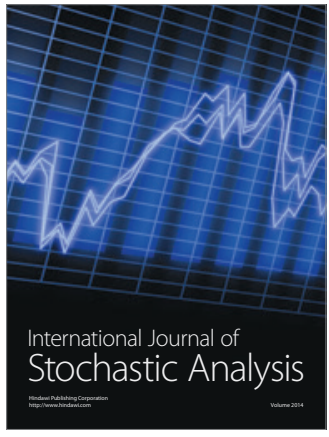

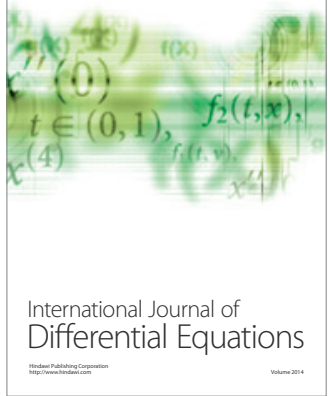
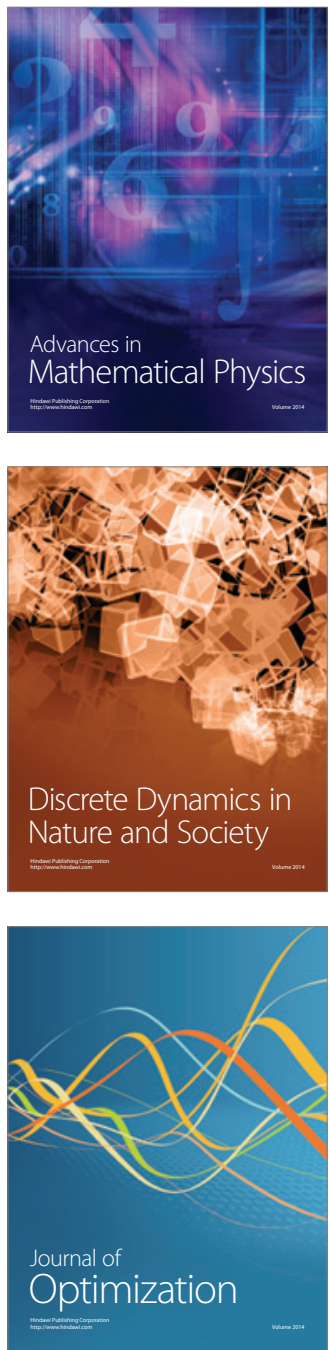